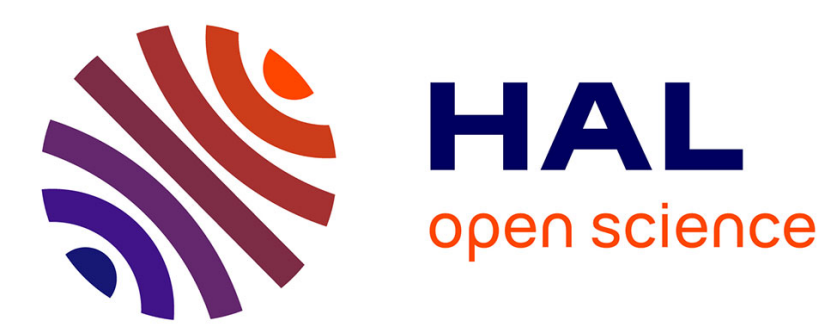

\title{
Generation of local magnetic field; application to VCSEL
} O. Gaiffe, S. Euphrasie, B. Cretin, P. Vairac

\section{To cite this version:}

O. Gaiffe, S. Euphrasie, B. Cretin, P. Vairac. Generation of local magnetic field; application to VCSEL. European Physical Journal: Applied Physics, 2009, 47 (1), pp.1-5. 10.1051/epjap/2009066 . hal-00480168

\section{HAL Id: hal-00480168 https://hal.science/hal-00480168}

Submitted on 3 May 2010

HAL is a multi-disciplinary open access archive for the deposit and dissemination of scientific research documents, whether they are published or not. The documents may come from teaching and research institutions in France or abroad, or from public or private research centers.
L'archive ouverte pluridisciplinaire HAL, est destinée au dépôt et à la diffusion de documents scientifiques de niveau recherche, publiés ou non, émanant des établissements d'enseignement et de recherche français ou étrangers, des laboratoires publics ou privés. 


\title{
Generation of local magnetic field; application to VCSEL
}

\author{
O. Gaiffe, S. Euphrasie, B. Cretin, and P. Vairac ${ }^{\text {a }}$
}

Institut FEMTO-ST, Université de Franche-Comté, CNRS, ENSMM, UTBM, 32 avenue de l'Observatoire F-25044 Besancon cedex - France

Received: date / Revised version: date

\begin{abstract}
In this article, we report on the simulation and realization of a magnetic circuit allowing to apply an axial magnetic field higher than 1 Tesla. The proposed electromagnet is used to investigate the influence of a magnetic field on the polarization behavior of a vertical-cavity-surface-emitting laser (VCSEL). A nonlinear polarization behavior can be experimentally observed for currents as low as the threshold current, provided that the magnetic field is strong enough.
\end{abstract}

PACS. 41.20.Gz Magnetostatics; magnetic shielding, magnetic induction, boundary-value problems 42.55.Px Semiconductor lasers; laser diodes - 42.25.Ja Polarization

\section{Introduction}

In the field of microsystems, the use of electric or magnetic fields for excitation or detection is common, and in most cases the required levels are relatively low. More particularly, for the generation of magnetic fields at this scale, microfabrication techniques are used to make micro-coils [1-4] with ferromagnetic cores. Yet, when it comes to generate a local magnetic field of about 1 Tesla, this type of techniques reach their limits and it is therefore necessary to develop a specific system in this context. We propose in this article the study and implementation of an electromagnet allowing for the generation of local magnetic fields up to 1 Tesla. We use this specific device as part of a study on the effect of an axial magnetic field on the polarization properties of a vertical-cavity surface-emitting laser (VCSEL). Several experiments actually show that the light generated by VCSELs exhibits a large variety of polarization behaviors [5-7]. Some papers discuss the effect of an axial magnetic field on the polarization behavior of $\mathrm{VC}$ SELs from a theoretical point of view, but there is only a single report on related experimental results [8]. The motivation of this paper is rooted in the progress concerning the understanding of this effect, a future step being to use these results in metrology, as the very long life time and the high coherence length (around $10 \mathrm{~m}$ ) of VCSELs are very promising in this area.

\section{Electromagnet}

\subsection{Presentation of the magnetic system}

We aim at an electromagnet producing a local magnetic field about 1 Tesla in the beam propagation direction. To

\footnotetext{
a e-mail: pascal.vairac@femto-st.fr
}

localize the magnetic field on the VCSEL, we designed a magnetic circuit based on 2500-turn coil with two conic poles. One of the poles was pierced to allow the VCSEL laser beam to go through. An important criterion to take into account to achieve such an electromagnet is that the laser beam has a divergence of about 20 degrees. Therefore, we have to adapt the dimensions of the hole in order not to block off the beam. The VCSEL was then mounted on a circuit board, along with the two conic poles, the distance between the two cones being set to $2 \mathrm{~mm}$ to be able to insert easily the VCSEL. The complete electromagnet is thus contained in a volume of $60 \times 60 \times 20 \mathrm{~mm}^{3}$. A schematic of the complete electromagnet design is displayed in Figure 1.

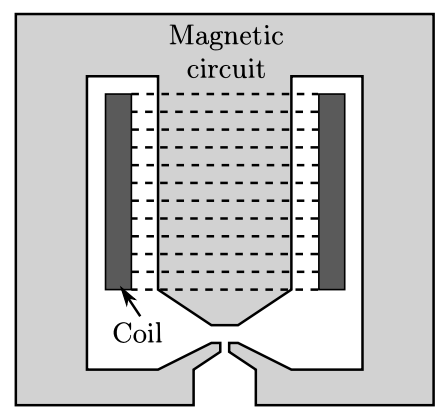

Fig. 1. Representation of the electromagnet

The exact dimensions of the electromagnet air gap are detailed in Figure 2. $h$ and $s$ are respectively the cone height and the tip radius. The radius of the base $B$ is set at $10 \mathrm{~mm}$. These three parameters condition the half-angle $\alpha$ of the cone. The second cone is pierced by a cylinder (radius $r$ and length of $1 \mathrm{~mm}$ ), by a cone (height $(h-1 \mathrm{~mm})$, 
cone tip radius $r$ and fixed base radius $R$ equal to $5 \mathrm{~mm}$ ) and finally by another cylinder (height $H$ and radius $R$ ). This geometry allows us to handle the issue of the VCSEL divergence. In the next paragraph, we will focus on the influence of the $h, s$ and $r$ parameters on the spatial distribution and intensity of the magnetic field $B_{z}$ in the air-gap.

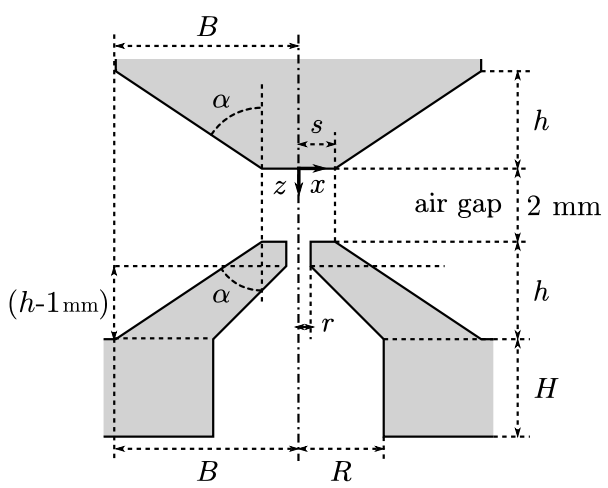

Fig. 2. Zoom of the magnetic circuit. $s$ is the radius of the two cone tips, $h$ is their height and $r$ is the hole radius.

\subsection{Magnetic simulation}

Simulations of this magnetostatic problem were carried out with CST ElectroMagnetic Studio. This commercial software is part of a suite based on the Finite Integration Technique (FIT) and the Perfect Boundary approximation $\mathrm{PBA}^{(T M)}[9-11]$. The FIT discretizes the integral form of Maxwell's equations, rather than the differential one, on a pair of dual interlaced discretization grids. The $\mathrm{PBA}^{(T M)}$ allows us to avoid the disadvantage of the staircase approximation of complex boundaries (for instance cone-shaped boundaries).

In our analysis, the current of the coil is noted $I_{b}$. We choose a maximum current $I_{b \max }$ of $500 \mathrm{~mA}$ to avoid temperature problems in the coil. The experiments reported here were performed at a value of $250 \mathrm{~mA}$. Figure 3 shows the intensity of the field $B_{z}$ along the $x$ axis (at $z=1 \mathrm{~mm}$ ) for two different shapes of magnetic circuit. The curve with open circles corresponds to a geometry composed of two cylinders ( $h=5 \mathrm{~mm}, s=10 \mathrm{~mm}$ and $r=1 \mathrm{~mm}$ ), while the curve with solid circles corresponds to a geometry composed of two cones $(h=5 \mathrm{~mm}, s=1.5 \mathrm{~mm}$ and $r=0.5 \mathrm{~mm}$ ).

As expected, the magnetic field along the $x$ axis is more localized for the geometry based on the cones than when using cylinders. There is no change in the magnetic field intensity $B_{z}$ at the center of the air gap. This can come from two opposing effects. On the one hand, the magnetic field lines are focused by the geometry of the cone, but on the other hand, there is more magnetic energy that escapes from the loop.

At the center of the air gap, the field value changes with the geometry of the cones as shown in Figure 4 which

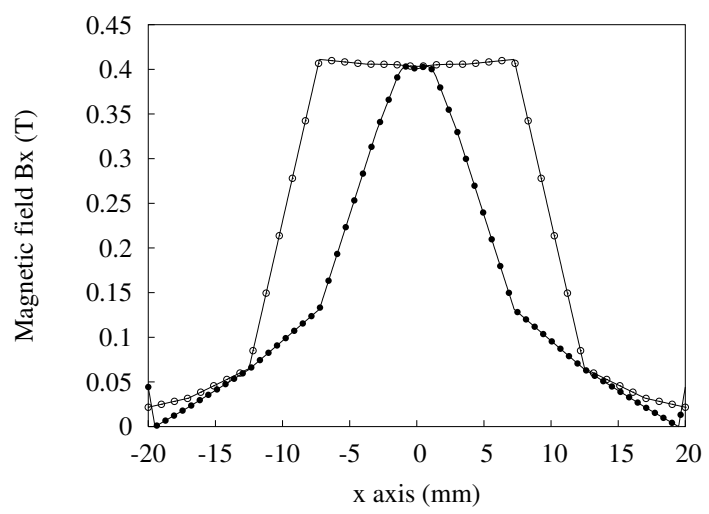

Fig. 3. Intensity of the magnetic field $B_{z}$ along the $x$ axis (at $z=1 \mathrm{~mm}$ ) for two different shapes of magnetic circuit. Open circles : two cylinders; solid circles : two cones.

shows the evolution of the field intensity $B_{z}$ on the $z$ axis for different values of $s$. The other parameters used are $h=5 \mathrm{~mm}$ and $r=0.4 \mathrm{~mm}$. The hole drilled to allow the beam output has a significant influence on $B_{z}$. It result in an asymmetric behavior (along the $z$ axis): the field $B_{z}$ is higher towards $z=0$ than towards $z=2 \mathrm{~mm}$. Conversely, when the variable $s$ is between 1 and $10 \mathrm{~mm}(s=10 \mathrm{~mm}$ corresponding to the case of cylinders), changes are negligible. For $s \geq 1 \mathrm{~mm}$, the magnetic field remains almost constant in the first half of the air-gap $(0 \geq z \geq 1 \mathrm{~mm})$. However, Figure 4 shows that the intensity of $B_{z}$ decreases when $z$ becomes larger than $1 \mathrm{~mm}$ and is even reduced to approximately $50 \%$ of its value at $z=2 \mathrm{~mm}$.

If we want to localise the field by reducing the value of $s(s=0.5 \mathrm{~mm})$, the behavior of the curve is different and the field is no longer constant in the first region of the air gap. At the center of the gap, there is also a decrease of $B_{z}$ by approximately $10 \%$. Using different values of $h$ (from 3 to $7 \mathrm{~mm}$ ) little change is observable.

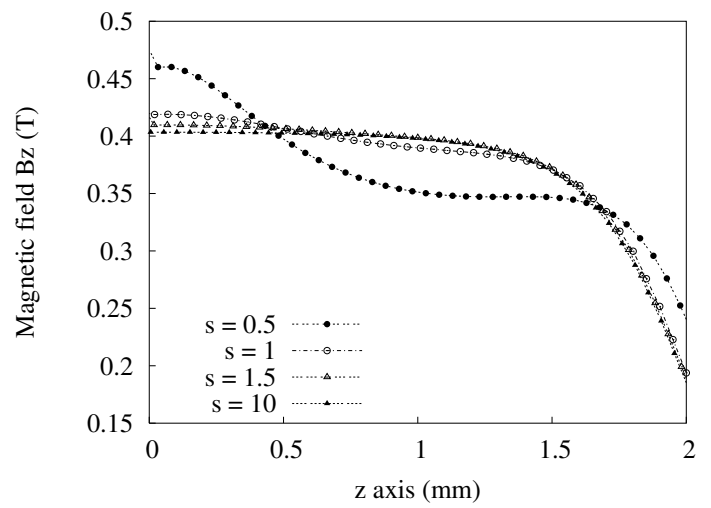

Fig. 4. Intensity of the magnetic field $B_{z}$ along the $z$ axis for increasing values of $s$ with $r=0.4 \mathrm{~mm}$ and $h=5 \mathrm{~mm}$.

Figure 5 shows the intensity of the field $B_{z}$ as a function of the hole radius $r$ for $s=0.5 \mathrm{~mm}$. We can see that there is little influence of $r$ on $B_{z}$ for $z<1.5 \mathrm{~mm}$. 
For $z>1.5 \mathrm{~mm}$, the field $B_{z}$ decreases with increasing $r$. As expected, when $r$ decreases, the curve tends to be symmetrical. However, when $r=0$ (no cylinder but still a cone-shaped hole) the magnetic field greatly increases near the second cone, showing that the hole concentrates the magnetic field $B_{z}$. Without the hole the curve is logically symmetrical. In the case of $s=1.5 \mathrm{~mm}$ (not represented here), a similar trend can be observed, but the decrease in $B_{z}$ for $r \geq 0.15 \mathrm{~mm}$ is not as pronounced.

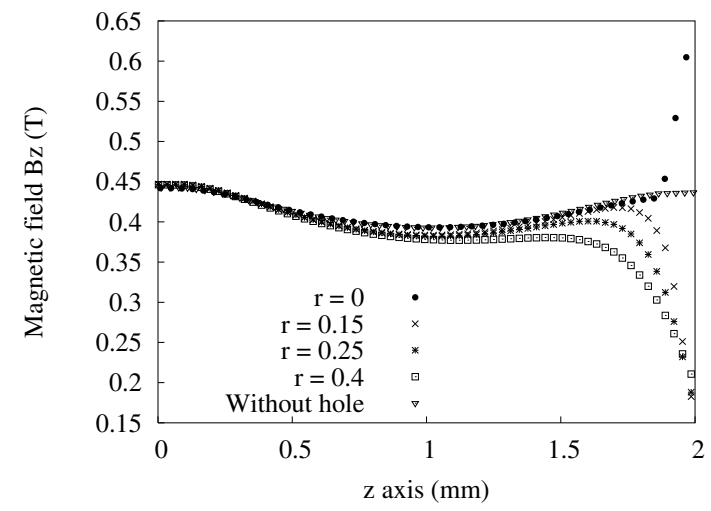

Fig. 5. Intensity of the magnetic field $B_{z}$ along the $z$ axis for increasing values of $r$ with $s=1.5 \mathrm{~mm}$.

To fabricate our electromagnet, we chose the following parameter values: $h=5 \mathrm{~mm}, s=1.5 \mathrm{~mm}$ and $r=0.5 \mathrm{~mm}$. As the effect of $\mathrm{h}$ is indeed negligible, its value was set to $5 \mathrm{~mm}$ in order to relieve the fabrication constraints. Setting $s=1.5 \mathrm{~mm}$ allows localizing the field while keeping it constant in the first half of the air gap. $r=0.5 \mathrm{~mm}$ has been chosen as it can be easily pierced. The VCSEL was then be placed at the centre of the gap.

With a maximum current $I_{b \max }=500 \mathrm{~mA}$, we get a magnetic field around $800 \mathrm{mT}$. This value is below the desired one, which is 1 Tesla. For that reason, we also used another design composed this time of two coils. The design is the same with the same parameters for the gap except for the magnetic circuit that is longer, hence allowing to place two coils. This has enabled us to exceed 1 Tesla. However, we observe that the magnetic field begins to saturate, as indicated in the following section.

\subsection{Comparison between simulations and experiments}

The measurement of the magnetic field $B_{z}$ is performed with a gaussmeter. The probe is $1.5 \mathrm{~mm}$ thick and the sensor is located in the middle of the probe. Figure 6 compares simulation results and experimental measurements by representing the evolution of $B_{z}$ as a function of current $I_{b}$. It shows a good agreement between the simulations and the measured field.

As mentioned earlier, to obtain a magnetic field $B_{z}$ larger than 1 Tesla, we made another electromagnet. With the same cone-shape but exhibiting an extra coil. The overall volume of the electromagnet is now $60 \times 100 \times 40 \mathrm{~mm}^{3}$

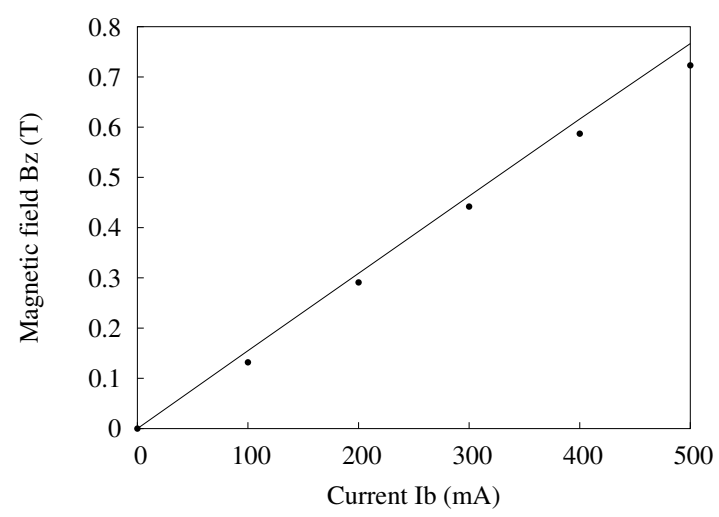

Fig. 6. Intensity of the magnetic field $B_{z}$ at $z=1 \mathrm{~mm}$ as a function of the coil current $I_{b}$ for a single coil. Solid curve: simulation results; Solid circles: experimental measurements.

which remains under the maximum desired dimension. Figure 7 compares the evolution of $B_{z}$ (with two coils) as a function of current $I_{b}$ for simulation results and experimental measurements. Once again, it shows a good agreement between the simulations and the measured field.

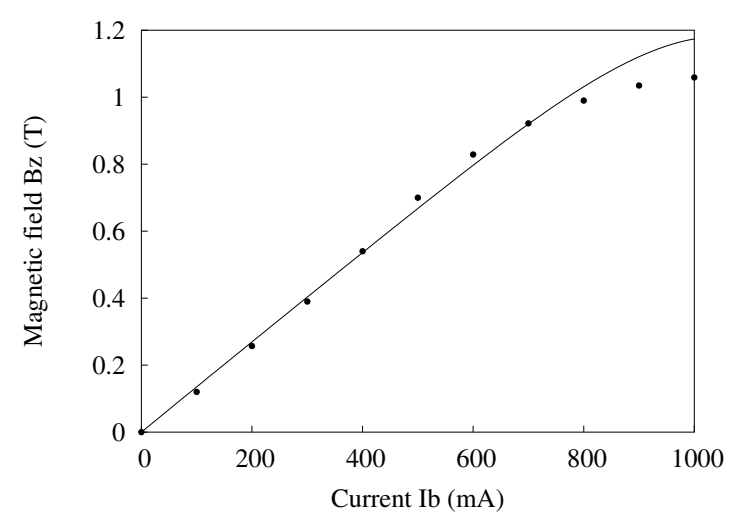

Fig. 7. Intensity of the magnetic field $B_{z}$ at $z=1 \mathrm{~mm}$ as a function of the coil current $I_{b}$ for two coils. Solid curve: simulation results; Solid circles: experimental measurements.

\section{Application to VCSEL}

\subsection{Background theory}

A VCSEL is composed of two distributed Bragg reflectors (DBR) separated by an active layer. The active region consists of one or several quantum wells allowing for laser light generation. A four-level model has been developed by San Miguel, Feng and Moloney [16] which takes into account the polarization of the laser field by including the spin sublevels of the conduction and valence bands of a semiconductor. Travagnin et al. [19] have extended the model to include the effect of a magnetic field. This , model treats the quantum-well gain medium as a four-level 
medium: right circularly polarized light $E_{+}$corresponds to the transition from $J_{z}=-1 / 2$ to $J_{z}=-3 / 2$ and left circularly polarized light $E_{-}$correspond to the transition from $J_{z}=1 / 2$ to $J_{z}=3 / 2$. The total population between the conduction and the valence band has a decay rate $\gamma_{p}$ due to spontaneous emission and $\gamma_{j}$ is the spin-mixing rate and account for the mixing of the population associated with right and left circularly polarized emission.

The global laser dynamics is described by the time evolution of the mean fields $E_{ \pm}$and of the carrier densities $D_{ \pm}$:

$$
\begin{aligned}
\dot{E_{ \pm}=} & -\kappa\left[(1-i \alpha)\left(1-D_{ \pm}\right) E_{ \pm}+\left(\epsilon_{l}+\imath \sigma_{l}\right) E_{\mp}\right. \\
& \left. \pm\left(\epsilon_{c}+\imath \sigma_{c}\right) E_{ \pm}\right] \\
\dot{D_{ \pm}}= & -\gamma_{p}\left[\left(1+\left|E_{ \pm}\right|^{2}\right) D_{ \pm}+\frac{\gamma_{j}}{\gamma_{p}}\left(D_{ \pm}-D_{\mp}\right)-(1+\beta)\right]
\end{aligned}
$$

where $\kappa$ is the cavity decay rate; $\epsilon_{l}$ and $\sigma_{l}$ are the linear amplitude and phase anisotropy; $\epsilon_{c}$ and $\sigma_{c}$ are the circular amplitude and phase anisotropy and $\beta$ is a normalized pumping parameter equal to zero at the lasing threshold.

To study the polarization properties of VCSEL generated light, it is useful to consider separately the amplitudes and phases of the fields, writing

$$
\begin{aligned}
& E_{+}=\left|E_{+}\right| \exp \left(-\imath \phi_{+}\right) \\
& E_{-}=\left|E_{-}\right| \exp \left(-\imath \phi_{-}\right)
\end{aligned}
$$

and to introduce the ellipticity angle

$$
\chi=\arctan \frac{\left|E_{+}\right|-\left|E_{-}\right|}{\left|E_{+}\right|+\left|E_{-}\right|}, \quad-\frac{\pi}{4}<\chi<\frac{\pi}{4}
$$

\subsection{Experimental setup}

We used a single-mode VCSEL operating at $850 \mathrm{~nm}$, distributed by Avalon Photonics. The total dimension of the VCSEL chip (electrical contacts included) is $230 \mu \mathrm{m} \times$ $280 \mu \mathrm{m}$. The electrical contact has a diameter of $80 \mu \mathrm{m}$. The VCSEL chips are glued with silver paste on a thin printed circuit board serving. The advantage of using these VCSELs lies in their large linewidth equal to $30 \mathrm{MHz}$ which allows their use in metrology.

In our experiment, the injection current $I$ is varied between $1.1 I_{t h r}$ and $1.8 I_{t h r}$ with $I_{t h r}=2.8 \mathrm{~mA}$. The resulting optical power hence ranges from 0.3 to $0.6 \mathrm{~mW}$. Under these conditions, the $\mathrm{TEM}_{00}$ mode is present. At zero magnetic field $\left(B_{z}=0 \mathrm{~T}\right)$, two peaks separated by about $\Delta v=12 \mathrm{GHz}$ are visible in the optical spectrum. This may be caused by a birefringence effect due to the cavity anisotropy of the VCSEL resulting from the manufacturing process $[12,13]$. The higher-frequency peak corresponds to the lasing mode and the lower-frequency peak corresponds to spontaneous emission in the orthogonal polarization mode.

The VCSEL is then put between the two poles of the electromagnet (see Fig. 8). A lens is positioned after the

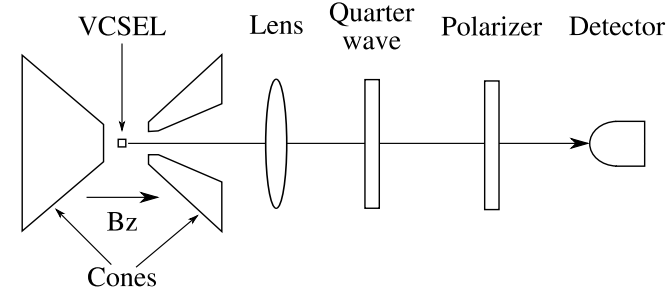

Fig. 8. Experimental set-up

electromagnet to collimate the laser beam. To analyse the ellipticity of the lasing mode, we use a combination of a quarter-wave plate and a polarizer. The polarizer is used to select a privileged axis to determine the behavior of the ellipticity angle as a function of the magnetic field.

\subsection{Experimental results: influence of the field on the VCSEL}

The higher-frequency peak, corresponding to the laser mode, has a polarization with a small ellipticity angle $2 \chi \leq 0.06 \mathrm{rad}$. Figure 9 shows the ellipticity angle for $I=1.1 I_{t h r}$ as a function of the magnetic field $B_{z}\left(I_{b}=100,200,300,400\right.$ and $500 \mathrm{~mA}$ ). The values of $B_{z}$ used here were obtained from the measurements reported in Figure 6.

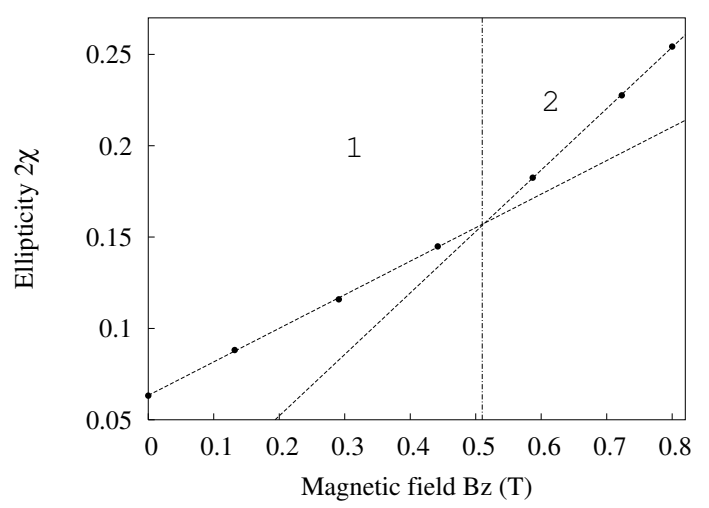

Fig. 9. Ellipticity angle $2 \chi$ as a function of magnetic field $B_{z}$ for injection current $I=1.1 I_{t h r}$.

The experimental curve can be divided into two distinct regions (above and below $B_{z}=500 \mathrm{mT}$ ). Both exhibit a linear behavior, though with different slope angles. The overall behavior is hence nonlinear. Under the same experimental conditions, Jansen Van Doorn et al. [8] also observe a linear variation of the ellipticity angle for $B_{z}<500 \mathrm{mT}$, i.e. in region 1 , but with a lower slope, meaning that the influence of the field $B_{z}$ on $\chi$ is less significant. The presented results can be explained by the value of the birefringence $(12 \mathrm{GHz})$ which is about twice as large. For stronger magnetic fields $\left(B_{z}>500 \mathrm{mT}\right)$, region 2 also shows another linear behavior with a higher slope.

A parameter usually employed in the literature to quantify the influence of a magnetic field on the polarization 
in VCSELs is the Verdet constant defined by:

$$
V=\frac{n}{c} \kappa \sigma_{l} \frac{\partial(2 \chi)}{\partial B}
$$

Taking $n=3.5$ for the average value of the refractive index and $\kappa=1.10^{12}[15,14]$, we obtain 0.808 rad.cm ${ }^{-1} . \mathrm{T}^{-1}$ for the first region and $1.479 \mathrm{rad} . \mathrm{cm}^{-1} . \mathrm{T}^{-1}$ for the second. As a comparison, Jansen et al. measured a Verdet constant of $0.97 \mathrm{rad} . \mathrm{cm}^{-1} . \mathrm{T}^{-1}$ for $B_{z}<500 \mathrm{mT}$, i.e. in region 1 . Similar experiments were carried out taking $I=1.8 I_{t h r}$. The "nonlinear" effect observed in Figure 9 is accentuated with increasing injection current $I$. Figure 10 shows the ellipticity angle for $I=1.8 I_{t h r}$ as a function of the magnetic field $B_{z}\left(I_{b}\right.$ ranging from 0 to $1000 \mathrm{~mA}$ with $100 \mathrm{~mA}$ steps). $B_{z}$ was obtained from the measurements reported in Figure 7. We observe that the bifurcation point, located between the two regions, moves towards the origin when the injected current increases. The reason is attributable to the saturation of $E_{+}$(right polarization) and $E_{-}$(left polarization). As in the previous case, for stronger magnetic fields, i.e. for $B_{z}>500 \mathrm{mT}$, the behavior remains linear, with a Verdet constant equal to $1.1 \mathrm{rad} . \mathrm{cm}^{-1} \cdot \mathrm{T}^{-1}$. However, the difference between the slopes at an injection current of $I\left(1.1 I_{t h r}\right.$ and $\left.I=1.8 I_{t h r}\right)$ respectively is approximately $25 \%$ for the highest values of $B_{z}$.

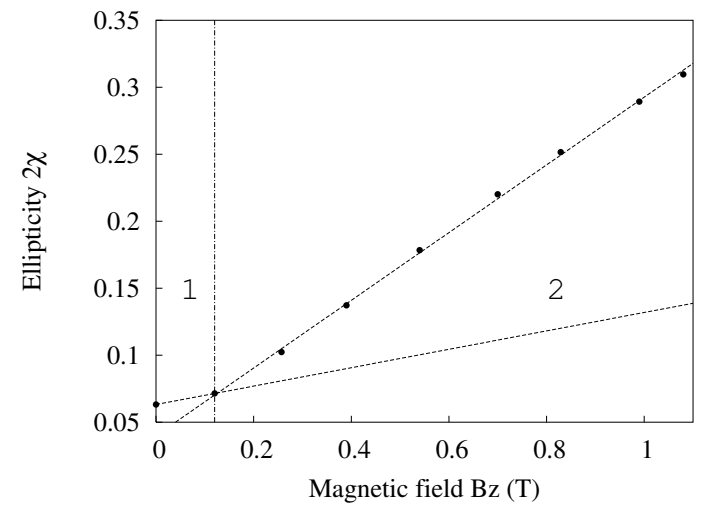

Fig. 10. Normalized ellipticity angle $2 \chi$ as a function of magnetic field $B$ for injection current $I=1.8 I_{t h r}$.

The results displayed in Figures 9 and 10 can allow us to retrieve the circular phase anisotropy. From the simplified model presented in [8]:

$$
\dot{E_{ \pm}}=\kappa\left( \pm \imath \sigma_{c}(B) E_{ \pm}-\imath \sigma_{l} E_{\mp}\right)
$$

with the eigenvalue $\tan (2 \chi)= \pm \sigma_{c} / \sigma_{l}$. It is therefore possible to determine $\sigma_{c}(B)$. The obtained values of the circular anisotropy can be used to evaluate the influence of the injection current on the ellipticity. $\sigma_{c}$ can be reinjected in the theoretical model (1), in order to determine the theoretical values of $\tan (2 \chi)$. To do this, we use the following parameters: $\sigma_{l}=\pi \kappa \Delta v=0.037, \gamma_{p}=10^{9} \mathrm{~s}^{-1}$, $\gamma_{j}=10^{11} \mathrm{~s}^{-1}[17,18], \alpha=3, \beta=0.1$ and $\epsilon_{l}=\epsilon_{c}=0$. For $I=1.1 I_{t h r}$, the difference between the theoretical and experimental results is about $6 \%$. For $I=1.8 I_{t h r}$ (ie $\beta=0.8$ ) the difference is greater and is about $30 \%$. These experimental investigations show the limits of the theoretical model in the case of high injection currents and demonstrate that an extended study is necessary to fully account for the observed nonlinear behavior.

\section{Conclusion}

We have described in this article the study, the simulation and the development of a specific electromagnet that can generate a local magnetic field higher than 1 Tesla. This magnetic circuit allowed to investigate the polarization behavior of VCSELs in the presence of an axial magnetic field. The experimental results showed that the dependence of this behavior on the magnetic field is non-linear, which can be observed for currents a low as the threshold current if the applied field is higher than 0.5 Tesla. This effect becomes more significant with increasing current. These experimental results are potentially interesting for the scientific community working in this area, since most of the reported works in this field remain limited to theoretical investigations. More generally, we believe that this study of a specific electromagnet for local scale generation of magnetic field can be useful for the characterization or excitation of MEMS.

The authors wish to express their thanks to S. Benchabane and D. Vernier for many stimulating discussions. This work is supported by La région de Franche-Comté of France.

\section{References}

1. M. Drndić, K. S. Johnson, J. H. Thywissen, M. Prentiss, and R. M. Westerveltb, Appl. Phys. Lett. 72, 2906 (1998)

2. C. S. Lee, H. Lee, and R. M. Westervelta, Appl. Phys. Lett. 79, 3308 (2001)

3. H. K. Kim, S. H. Hong, B. C. Kim, J. S. Hwang, S. M. Seong, T. H. Park, S. W. Hwang and D. Ahn, Jpn. J. Appl. Phys. 43, 2054 (2004)

4. H. Lee, A. M. Purdon, and R. M. Westervelta, Appl. Phys. Lett. 85, 1063 (2004)

5. H. E. Li, K. Iga, Vertical-Cavity Surface-Emitting Laser Devices (Springer, New York, 2003).

6. A. K. Jansen van Doorn, M. P. van Exter, and J. P. Woerdman, Appl. Phys. Lett. 69, 3635 (1996)

7. K. Panajotov, B. Nagler, G. Verschaffelt, A. Georgievski, H. Thienpont, J. Danckaert and I. Veretennicoff, Appl. Phys. Lett. 77, 1590 (2000)

8. A. K. Jansen van Doorn, M. P. van Exter, M. Travagnin and J. P. Woerdman, Optics Comm. 133, 252 (1997)

9. B. Krietenstein, R. Schuhmann, P. Thoma and T. Weiland, in Proceedings of LINAC'98, (1998), p. 860

10. H. Spachmann and U. Becker, Nucl. Instrum. Methods Phys. Res. Sect. A-Accel. Spectrom. Dect. Assoc. Equip. 558, 50 (2006)

11. L. Lau, I. Munteanu, F. Demming and M. Walter, in Proceedings of Asia-Pacific Conference on Applied Electromagnetics, APACE 2005 (2005), p. 8 
12. K. D. Choquette, K. L. Lear, R. E. Leibenguth and M. T. Asom, Appl. Phys. Lett. 21, 2767 (1994)

13. A. K. Jansen van Doorn, M. P. van Exter and J. P. Woerdman, Electron. Lett. 30, 1941 (1994)

14. J. W. Scott, B. J. Thibeault, C. J. Mahon, L. A. Coldren and F. H. Petters, Appl. Phys. Lett. 65, 1483 (1994)

15. D. Tauber, G. Wang, R. S. Geels, J. C. Bowers and L. A. Coldren, Appl. Phys. Lett. 62, 325 (1993)

16. M. San Miguel, Q. Feng and J. V. Moloney, Phys. Rev. A 52, 1728 (1995)

17. R. Ferreira and G. Bastard, Phys. Rev. B 43, 9687 (1991)

18. M. Z. Maialle, E. A. de Andrada e Silva and L. J. Sham, Phys. Rev. B 47, 15776 (1993)

19. M. Travagnin, M. P. van Exter, A. K. Jansen van Doorn, and J. P. Woerdman, Phys. Rev. A 54, 1647 (1996) 